\title{
THE AAVSO DATABASE OF VARIABLE
}

\section{STAR OBSERVATIONS}

\author{
J. R. PERCY \\ Erindale Campus, University of Toronto, Mississauga, Ontario, Canada L5L 1 C6 \\ and \\ J. A. MATTEI \\ Director: AAVSO 25 Birch Street, Cambridge, MA 02138-1205, USA
}

The American Association of Variable Star Observers (AAVSO) is the largest organization of variable star observers in the world, with members in 42 countries. The purpose of the AAVSO is to coordinate variable star observing, done primarily by amateur astronomers, evaluate the accuracy of these observations, compile, process and publish them, and make them available to researchers and educators around the world. Over 6.5 million observations of variable stars have been complied since the AAVSO was founded in 1911. Those since about 1960 are in computerized form, and it is intended to have all observations in this form within the next year. About 250,000 observations are submitted to and archived by the AAVSO each year, over half of them from outside the USA. Most of the observations are visual, but there is also an active photoelectric program which concentrates on semi-regular and irregular stars such as small-amplitude red variables. In 1990, over 200 requests for AAVSO data and services were received from researchers and educators; this number has increased by a factor of 10 in the last two decades.

AAVSO services are sought by astronomers (a) to receive real-time, upto-date information on unusual stellar behaviour, (b) to assist scheduling and execution of variable star observing programs using Earth-based large telescopes, and instruments aboard satellites; (c) to request simultaneous optical coverage of stars being studied during Earth-based or satellite observing programs, and immediate notification of their activity; (d) to correlate optical data with data obtained using other techniques (photometry, spectroscopy, polarimetry), and at other wavelengths from gamma-rays to radio waves; (e) to carry out collaborative long-term data analyses of the behaviour of (particularly) large-amplitude variable stars; and (f) to assist educators in setting up observing projects, and students in carrying them out.

For many hundreds of stars, the AAVSO has almost continuous visual observations, using consistent sets of comparison stars, and extending over many decades. These can be used to study long-term changes in period, amplitude, mean magnitude, and light curve shape in large-amplitude stars 
such as Mira variables (Percy et al. 1990). Although the precision of a single visual observation is relatively low (typically, 0.2 to 0.3 magnitude), useful studies of smaller-amplitude variables can be done by Fourier analyzing the observations, grouping them into means, or otherwise processing them so as to extract the maximum amount of information (e.g. the study of Rho Cas by Percy et al. 1985). AAVSO photoelectric observations, which for some stars now extend over almost a decade, are well-suited for studying longterm changes in smaller-amplitude variables, and for distinguishing between regular and irregular behaviour (e.g. the study of EU Del by Percy et al. 1989).

AAVSO visual observations have also been used to search for chaotic behaviour in Mira stars (Cannizzo et at. 1990), the cataclysmic variable SS Cyg (Cannizzo and Goodings 1988; Hempelmann and Kurths 1990), and the RV Tauri star R Sct (Veldhuizen and Percy 1989). There are several dozen RV Tauri and SRd variables in the AAVSO visual and photoelectric observing programs. These observations, if suitably processed and analyzed, may be extremely useful for studying the nature of the irregularity in these stars.

To request AAVSO data and services, please contact the Director at the address above, or by e-mail at AAVSO@CFA8.BITNET.

\section{References}

Cannizzo, J. K. and Goodings, D. A.: 1988, Astrophysical Journal 334, L31.

Cannizzo, J. K., Goodings, D. A. and Mattei, J. A.: 1990, Astrophysical Journal 357, 235.

Hempelmann, A. and Kurths, J.: 1990, Astronomy and Astrophysics 232, 356.

Percy, J. R., Colivas, T., Sloan, W. B. and Mattei, J. A.: 1990, in Confrontation between Stellar Pulsation and Evolution, eds. C. Cacciari and G. Clementini (ASP Conference Series \#11).

Percy, J. R., Fabro, V. A. and Keith, D. W.: 1985, JAAVSO 14, 1.

Percy, J. R., Landis, H. J. and Milton, R. E.: 1989, Publications of the ASP 101, 893.

Veldhuizen, T. and Percy, J. R.: 1989, JAAVSO 18, 97. 